\title{
Minimize electricity generation cost for large scale wind- thermal systems considering prohibited operating zone and power reserve constraints
}

\author{
Phan Nguyen Vinh', Bach Hoang Dinh ${ }^{2}$, Van-Duc Phan ${ }^{3}$, Hung Duc Nguyen ${ }^{4}$, Thang Trung Nguyen ${ }^{5}$ \\ ${ }^{1}$ Faculty of Cinema and Television, The University of Theatre and Cinema Ho Chi Minh City, Ho Chi Minh City, \\ Vietnam \\ ${ }^{2,5}$ Power System Optimization Research Group, Faculty of Electrical and Electronics Engineering, Ton Duc Thang \\ University, Ho Chi Minh City, Vietnam \\ ${ }^{3}$ Faculty of Automobile Technology, Van Lang University, Ho Chi Minh City, Vietnam \\ ${ }^{4}$ Faculty of Electrical and Electronics Engineering, Ho Chi Minh City University of Technology, \\ Vietnam National University, Ho Chi Minh City, Vietnam
}

\section{Article Info}

Article history:

Received Jun 10, 2020

Revised Sep 29, 2020

Accepted Oct 10, 2020

\section{Keywords:}

Combined systems

Fuel cost

Power reserve

Prohibited working zone

Thermal power plants

Wind power plants

\begin{abstract}
Wind power plants (WPs) play a very important role in the power systems because thermal power plants (TPs) suffers from shortcomings of expensive cost and limited fossil fuels. As compared to other renewable energies, WPs are more effective because it can produce electricity all a day from the morning to the evening. Consequently, this paper integrates the optimal power generation of TPs and WPs to absolutely exploit the energy from WPs and reduce the total electricity generation cost of TPs. The target can be reached by employing a proposed method, called one evaluation-based cuckoo search algorithm (OEB-CSA), which is developed from cuckoo search algorithm (CSA). In addition, conventional particle swarm optimization (PSO) is also implemented for comparison. Two test systems with thirty TPs considering prohibited working zone and power reserve constraints are employed. The first system has one wind power plant (WP) while the second one has two WPs. The result comparisons indicate that OEBCSA can be the best method for the combined systems with WPs and TPs.
\end{abstract}

This is an open access article under the CC BY-SA license.

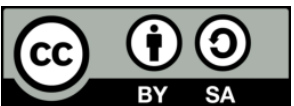

\section{Corresponding Author:}

Thang Trung Nguyen

Power System Optimization Research Group, Faculty of Electrical and Electronics Engineering

Ton Duc Thang University, 19 Nguyen Huu Tho street, Tan Phong ward, District 7

Ho Chi Minh City, Vietnam

Email: nguyentrungthang@tdtu.edu.vn

\section{INTRODUCTION}

Optimal operation of power systems plays a very important role in reaching high economy and stable operation status of all electric components such as transmission lines, distribution lines, transformers, generators, and capacitors [1, 2]. Among different power plants such as thermal power plants (TPs), hydropower plants (HPs), wind power plants (WPs), solar thermal power plants (SPs) and photovoltaic power plants (PPs), TPs are consuming huge amount of fossil fuels (FFs) with very high cost. Furthermore, FFs will be distinct in the future and electric energy power safety will not be warranted. So, the integration of using both TPs and other power plants with smaller cost should be implemented, especially with WPs, SPs and PPs. Currently, the core task of operators in TPs is to allocate the most optimal power generation to reach the lowest electric generation cost by burning FFs as much as possible [3-5] 
The core task of reaching the lowest fossil fuel cost (FFC) from TPs has been concerned and attracted a huge number of researchers so far. This task and the whole problem of fossil fuel cost reduction are described in detail in Economic load dispatch (ELD) [6-8]. The problem can be mathematically formulated by presenting objective function and a constraint set in which FFC reduction is objective while generation limits of generators in TPs and balance of active power between supply side and consuming side are regarded as major constraints. This is the simplest model of ELD in power system optimization operation. For more complicated ELD problem, prohibited working zones, ramp rate limits of generation as well as power reserve demand are taken into consideration [9-12]

Thanks to the development of computation algorithms, ELD problems without and with the most complicated constraints have been solved successfully. The applied method groups are classified into derivative-based method group [11-13] and population-based method group [14-20]. The comer is low performance and its applications are not wide such as Lagrange algorithm [11], Newton algorithm [12], and gradient search [13]. On the contrary, the latter is highly effective and applied more widely and successfully such as modified moth swarm algorithm (MMSA) [14], stochastic fractal search algorithm (SFSA) [15], Bee optimization algorithm (BOA) [16-17], teaching-learning algorithm (TLA) [18], crow algorithm (CA) [19] and hybrid algorithm (HA) [20]. However, there was no consideration of renewable energies in the studies such as WPs, SPs and PPs. Nowadays, WPs can generate a high power and provide a huge electricity power energy to power systems. The generation cooperation of WPs and TPs has been studied and presented in different works [21, 22] for cost reduction in TPs. Uncertainty characteristic of wind velocity (WV) was studied and represented as a probability function [21]. WV was uncertain and power generation of wind turbines was also uncertain. For another case with the assumption of certainty of WV, TPs are in charge of producing the remaining power of load demand. PSO and bat optimizer (BO) were used as two main tools in allocating optimal generation of TPs and WPs [22].

In this paper, the electricity generation integration of WPs and TPs is studied for the core task of electricity cost reduction. The duty of cost reduction and constraint satisfactory is accomplished by using PSO [23-24], cuckoo search algorithm (CSA) [25, 26] and one evaluation-based CSA (OEB-CSA). Among the methods, OEB-CSA is first developed in the paper. The three methods are run on two different systems, the fifteen-TPs and one-Wind power plant (WP) system, and the thirty-TPs and two-WPs system. The novelty of the paper is as follows:

- Propose a new algorithm, called OEB-CSA

- Propose the integration of WPs and TPs considering prohibited working zone (PWZ) and power reserve (PR) constraints

The main contribution of the paper is as follows:

- Introduce a better algorithm than CSA and PSO

- Reach low cost for TPs

- Provide enough power and power reserve to system

\section{OPTIMAL LOAD DISPATCH PROBLEM DESCRIPTION}

\subsection{Electric generation cost and objective}

In ELD problem, electric generation cost only considers cost of fossil fuel meanwhile other costs regarding humanity and benefit are neglected. The consideration is because fossil fuel is directly related to power generation. The cost of power generation is a second order function with respect to power generation is being as (1) [1]:

$$
F F C_{k}=\alpha_{k} P_{k}^{2}+\lambda_{k} P_{k}+\delta_{k}
$$

where $F F C_{k}$ is the fossil fuel cost of the kth TP; $\delta_{k}, \lambda_{k}, \alpha_{\mathrm{k}}$ are coefficients, which are obtained by experiment; $\mathrm{Pk}$ is the power generation of the $k t h \mathrm{TP}$.

As considering a number of available TPs $\left(N_{T P S}\right)$ providing electricity to loads, total cost (TC) of all TPs is an objective function that needs to be minimized as shown in (2) [27]:

$$
\text { Minimize } F C=\sum_{k=1}^{N_{T P_{s}}} F F C_{k}
$$

The objective function is subject to the following constraints. 


\subsection{Constraints}

\subsubsection{Balance constraint}

As considering both TPs and WPs as two power sources supplying electricity to loads, the total power of the plants must be equal to load demand with assumption of neglecting power loss in transmission lines. This is power balance and presented by (3):

$$
\sum_{k=1}^{N_{T P_{s}}} P_{k}+\sum_{m=1}^{N_{W P_{s}}} P_{w m}-P_{\text {Loads }}=0
$$

where $P_{W m}$ is the power generation of the mth WP; $N_{W P S}$ is the number of WPs; and $P_{\text {Loads }}$ is the demand of all loads in the system.

\subsubsection{Power generation limits}

The generator in TPs as well as WPs must be run within its capacity, which is higher than the minimum generation for the economic purpose and not higher than rated power for the safety purpose. The limits are seriously considered in ELD problem and the two following inequalities must be satisfied.

$$
\begin{aligned}
& P_{k \text { min }} \leq P_{k} \leq P_{k \text { max }} \\
& P_{w m \text { min }} \leq P_{w m} \leq P_{w m \text { max }}
\end{aligned}
$$

Where $P_{k \min }$ and $P_{k \max }$ are the minimum and maximum generation of the $k$ th TP; and $P_{\text {wmmin }}$ and $P_{\text {wmmax }}$ are the minimum and maximum generation of the $m t h \mathrm{WP}$.

The $P_{w m}$ is calculated by using the formula (6):

$$
P_{w m}=\left\{\begin{array}{lc}
0 & \text { if } W V \leq W V i \\
P_{\text {wm, rate }} & \frac{W V-W V i}{W V r-W V i} \quad \text { if } W V i \leq W V \leq W V r \\
P_{\text {wm, rate }} & \text { if } W V r \leq W V \leq W V o \\
0 & \text { else }
\end{array}\right.
$$

where $P_{w m}$ and $P_{w m, \text { rate }}$ are generation and rated generation of the $m t h \mathrm{WP} ; W V$ and $W V r$, are speed and rated speed of wind at the mth WP; and $W V i$ and $W V o$ are the lowest and highest velocity of wind for power generation.

\subsubsection{Prohibited working zone (PWZ)}

This is the zone that generators cannot be run due to the safety constraint. Generators of TPs have to be run outside the zone although the power in the zones can reduce the FFC for the kth TP. In order to satisfy the constraint, power generation of the $k t h$ TP must satisfy the formula (7):

$$
P_{k} \in\left\{\begin{array}{l}
P_{k \min } \leq P_{k} \leq P_{k 1}^{l} \\
P_{k z-1}^{u} \leq P_{k} \leq P_{k z}^{l} ; z=2, \ldots . ., N_{k P W Z s} \\
P_{k N_{k P W z}^{u}}^{u} \leq P_{N_{k P W z}} \leq P_{k \max }
\end{array}\right.
$$

where $N_{k P W Z s}$ is the number of prohibited working zones (PWZs) of the kth TP; $P_{k z-1}^{u}$ is the upper bound of the $(z-1)$ th PWZ; and $P_{k z}^{l}$ is the lower bound of the $z$ th PWZ.

\subsubsection{Power reserve limit}

In order to warrantee loads are always supplied enough power all the time even one largest TP stops working suddenly, power reserve must be recalled from all available TPs in the system. As considering power reserve limit, each TP cannot work at the maximum power generation and the remaining power from all available TPs must be at least equal to the power reserve required by the power system. The constraint is shown in (8): 


$$
\sum_{k=1}^{N_{T P S}} R P_{k} \geq R P
$$

where $R P_{k}$ is power reserve of the kth TP; and $R P$ is the power reserve of the system.

$\mathrm{RP}$ is a known factor meanwhile $R P_{k}$ must be determined dependent on the power generation and the maximum power reserve as (7):

$$
R P_{k}= \begin{cases}P_{k \max }-P_{k} & \text { if } R P_{k \max }>\left(P_{k \max }-P_{k}\right) \\ R P_{k \max } & \text { else }\end{cases}
$$

where $R P_{k m a x}$ is the maximum power reserve of the $k t h \mathrm{TP}$.

\section{THE PROPOSED ALGORITHM}

\subsection{Cuckoo search algorithm}

\subsubsection{Exploration mechanism}

This mechanism explores large search space based on Lévy flights distribution with infinite limits. Thank to the mechanism, each old solution $S_{x}$ is newly updated as shown in the formula (10) [28]:

$$
S_{x, \text { new }}=S_{x}+r . \operatorname{Levy}\left(S_{\text {best }}-S_{x}\right) ; x=1, \ldots, \text { Po }
$$

where $r$ is positive scaling factor within 0 and 1; Levy is Lévy distribution; $S_{\text {best }}$ is the best solution of the population; $P o$ is the population size; and $S_{x, \text { new }}$ is the new $x t h$ solution.

\subsubsection{Exploitation mechanism}

On the contrary to exploration mechanism, exploitation mechanism focuses on small search space nearby each old solution. A step size far away from each old solution is formed based on two random solutions $S_{1}$ and $S_{2}$; however, not every old solution is newly updated. This is the second difference between exploitation and exploration mechanisms. Each old solution $S_{x}$ is produced a random number $r_{x}$ and this factor is compared to a mutation factor $F_{M u}$, which is randomly generated within 0 and 1 . The exploitation mechanism is shown in (11) [29]:

$$
S_{x, \text { new }}= \begin{cases}S_{x}+r d .\left(S_{1}-S_{2}\right) & \text { if } r_{x}<F_{M u} \\ S_{x} & \text { otherwise }\end{cases}
$$

where $r d$ is a randomly generated number in the range of 0 and 1; and $S_{1}$ and $S_{2}$ are two solutions randomly selected from the candidate population.

\subsection{One evaluation-based cuckoo search algorithm (OEB-CSA)}

As shown in section 3.1, CSA includes two mechanisms of producing new solutions. So, it takes CSA long time to implement a high number of solution evaluations, namely $(2 \times P o)$ evaluations. This seems to be the main restriction of CSA and it should be tackled. In this paper, OEB-CSA is proposed to shorten the computation time of producing new solutions and implementing new solution evaluations. The proposed method is implemented for finding the best solution until the computation iteration (It) is equal to the maximum iteration MaxIter as shown in Figure 1 of Appendix.

\section{NUMERICAL RESULTS}

In this section, three implemented methods including PSO, CSA and OEB-CSA solve two systems for reducing total cost of all TPs in the integrated systems. Each applied method is run for getting candidate solutions of 50 trials on Matlab and a computer- 2.2 GHz and 4.0 GB-RAM. The detail of simulation result is presented in the two following sections. In the first system, the first WP in [30] with rated wind speed and rated electric power of $15 \mathrm{~m} / \mathrm{s}$ and $120 \mathrm{MW}$ is considered. In the second system, two WPs in [30] are integrated with 30 TPs. The first WP is the same that in the first system while the second WP has rated wind speed and rated electric power of $15 \mathrm{~m} / \mathrm{s}$ and $80 \mathrm{MW}$. In order to run the three applied methods, the 
maximum iteration is selected to be 500 but population is selected not to be the same for the three methods, 50 for CSA and OEB-CSA, and 100 for PSO.

\subsection{Results from the first system}

In the first system, thirty TPs [4] and one WP [30] supply electricity to load of $5300 \mathrm{MW}$ and power reserve of $400 \mathrm{MW}$. The result summary is shown in Figure 2 and the detail of 50 runs is shown in Figure 3. Figure 2 sees OEB-CSA is the best method with the same minimum cost with CSA and much less minimum cost than PSO. Furthermore, OEB-CSA can reach better mean cost and maximum cost than both CSA and PSO. Figure 3 reveals the strong stability of OEB-CSA since all runs of the method are lied on a line while those of PSO and CSA have fluctuations, especially PSO with very high fluctuations. The more promising costs indicate that OEB-CSA is more robust than the two methods in finding the best solution and the search stability capability.

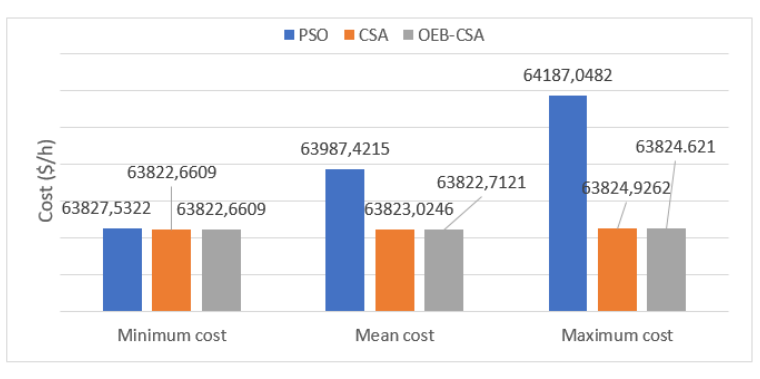

Figure 2. Summary of results from 50 runs for system 1

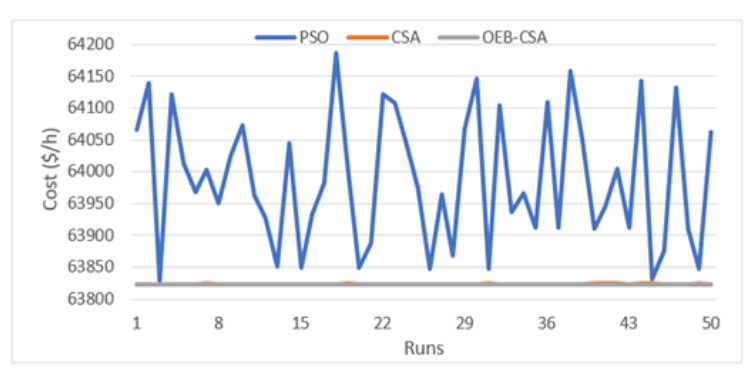

Figure 3. Cost of 50 runs for system 1

\subsection{Results from the second system}

In the second system, thirty TPs [4] and two WPs [30] are in charge of providing electricity to load and power reserve of $5300 \mathrm{MW}$ and $400 \mathrm{MW}$, respectively. Summary and detail of 50 runs are plotted in Figures 4 and 5, respectively. Figure 4 shows that OEB-CSA can get the lowest minimum, mean and maximum cost whereas Figure 5 presents very small fluctuations of 50 runs from OEB-CSA. But the fluctuations from PSO and CSA are more significant, especially PSO. The results see that OEB-CSA is the best in searching the best solution and stabilize the search process. The optimal generation of the two systems is shown in Table A1 of Appendix.

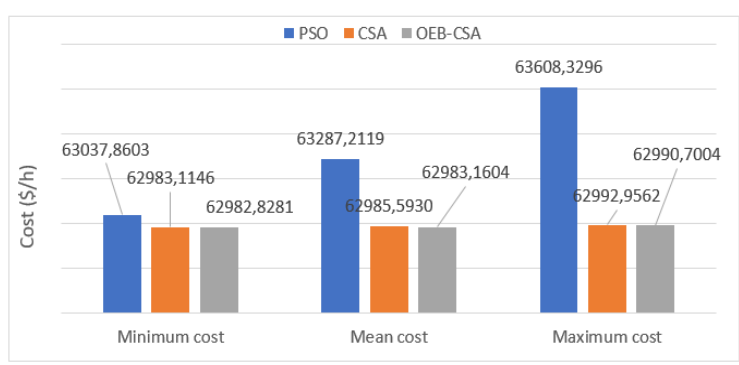

Figure 4. Summary of results from 50 runs for system 2

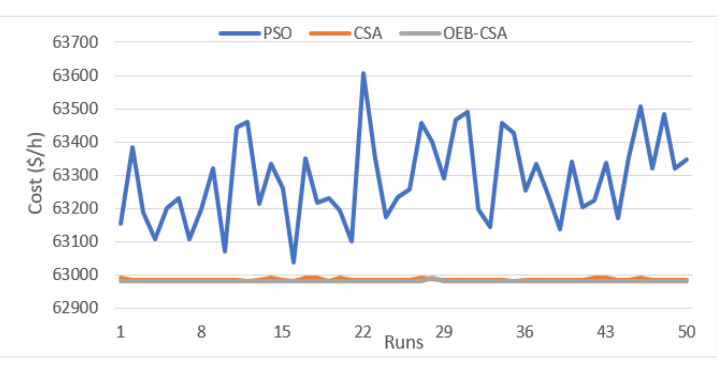

Figure 5. Cost of 50 runs for system 2

\section{CONCLUSION}

In this paper, three methods such as PSO, CSA and OEB-CSA have been implemented for two combined systems with TPs and WPs. In the two systems, thirty TPs have considered complicated constraints such as prohibited working zone and power reserve meanwhile one WP and two WPs have been joined to provide electricity to loads in system 1 and system 2, respectively. Each method has been run fifty times for reaching results such as minimum, average and maximum fuel cost for comparison. In addition, the stability of search of the three methods was also investigated by collecting standard deviation of fifty runs. PSO was the worst method with higher costs than CSA and OEB-CSA for two systems while OEB-CSA could reach 
the same or better minimum cost than CSA but better stability than CSA. Consequently, OEB-CSA is suggested a promising solution method for solving problem with the generation integration of TPs and WPs.

\section{APPENDIX}

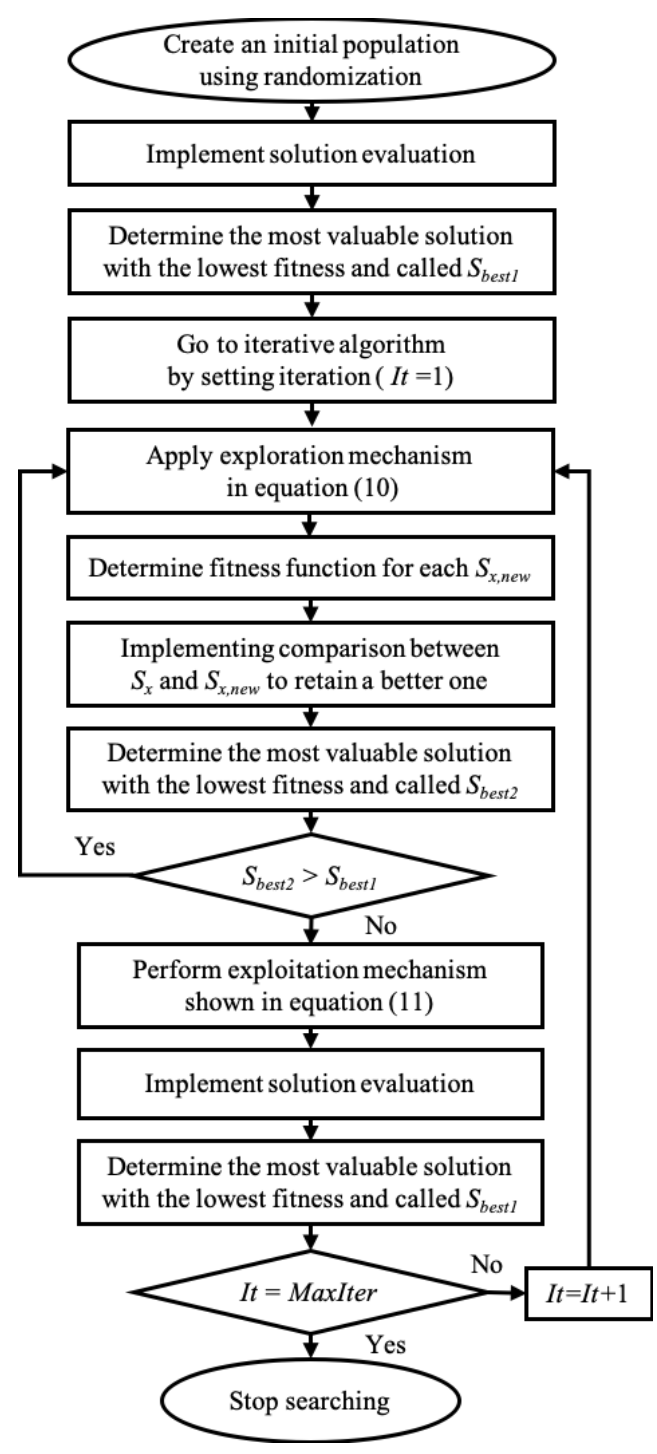

Figure 1. The flowchart of solving an optimization problem using OEB-CSA

Table A1. Optimal power generation obbtained by the OEB-CSA for the two systems

\begin{tabular}{ccccccccc}
\hline$P_{k}(\mathrm{MW})$ & System 1 & System 2 & $P_{k}(\mathrm{MW})$ & System 1 & System 2 & $P_{k}(\mathrm{MW})$ & System 1 & System 2 \\
\hline 1 & 455.0 & 455.0 & 11 & 20 & 20 & 21 & 460 & 459.9506 \\
2 & 455.0 & 455.0 & 12 & 55.0232 & 55.0055 & 22 & 465 & 464.9988 \\
3 & 130.0 & 130.0 & 13 & 25 & 25 & 23 & 60 & 60 \\
4 & 130.0 & 130.0 & 14 & 15 & 15 & 24 & 25 & 25 \\
5 & 260.0 & 214.9 & 15 & 15 & 15.0003 & 25 & 20 & 20 \\
6 & 460.0 & 460.0 & 16 & 455 & 454.9968 & 26 & 20 & 20.0021 \\
7 & 465.0 & 465.0 & 17 & 455 & 455 & 27 & 55.0568 & 51.8342 \\
8 & 60.0 & 60.0 & 18 & 130 & 130 & 28 & 25 & 25 \\
9 & 25.0 & 25.0 & 19 & 130 & 130 & 29 & 15 & 15 \\
10 & 20.0 & 20.0 & 20 & 259.9285 & 228.337 & 30 & 15 & 15 \\
\hline
\end{tabular}




\section{REFERENCES}

[1] I. N. Trivedi, P. Jangir, M. Bhoye, N. Jangir, "An economic load dispatch and multiple environmental dispatch problem solution with microgrids using interior search algorithm," Neural Computing and Applications, vol. 30, no. 7, pp. 2173-2189, 2018

[2] D. Das, A. Bhattacharya, R. N. Ray, "Dragonfly Algorithm for solving probabilistic Economic Load Dispatch problems," Neural Computing and Applications, vol. 32, pp. 3029-3045, 2020.

[3] K. Gnawali, K. H. Han, Z. W.Geem, K. S. Jun, K. T. Yum, "Economic Dispatch Optimization of Multi-Water Resources: A Case Study of an Island in South Korea," Sustainability, vol. 11, no. 21, p. 5964, 2019.

[4] C. T. Su, C. L. Chiang, "Nonconvex power economic dispatch by improved genetic algorithm with multiplier updating method," Electric Power Components and Systems, vol. 32, no. 3, pp. 257-273, 2004.

[5] M. A. Al-Betar, M. A. Awadallah, M. M. Krishan, "A non-convex economic load dispatch problem with valve loading effect using a hybrid grey wolf optimizer," Neural Computing and Applications, vol. 32, pp. 12127-12154, 2020.

[6] V. K. Kamboj, S. K. Bath, J. S. Dhillon, "Solution of non-convex economic load dispatch problem using Grey Wolf Optimizer," Neural Computing and Applications, vol. 27, no. 5, pp. 1301-1316, 2016.

[7] Q. Xu, Y. Ding, A. Zheng, "An optimal dispatch model of wind-integrated power system considering demand response and reliability," Sustainability, vol. 9, no. 5, pp. 758, 2017.

[8] D. Guo, J. Yu, M. Ban, "Security-constrained unit commitment considering differentiated regional air pollutant intensity," Sustainability, vol. 9, no. 5, p. 758, 2017.

[9] K. Mahmoud, M. Abdel-Nasser, E. Mustafa, Z. M Ali, "Improved Salp-Swarm Optimizer and Accurate Forecasting Model for Dynamic Economic Dispatch in Sustainable Power Systems,” Sustainability, vol. 12, no. 2, p. 576, 2020.

[10] V. K. Kamboj, A. Bhadoria, S. K. Bath, "Solution of non-convex economic load dispatch problem for small-scale power systems using ant lion optimizer," Neural Computing and Applications, vol. 28, no. 8, pp. 2181-2192, 2017.

[11] J. Zhou, "Solving economic dispatch problem with piecewise quadratic cost functions using Lagrange multiplier theory," In Proc. 3rd ICCTD, pp. 25-27, 2011.

[12] N. Kaur, I. Singh, "Economic Dispatch Scheduling using Classical and Newton Raphson Method," International Journal of Engineering and Management Research (IJEMR), vol. 5, no. 3, pp. 711-716, 2015.

[13] S. Ray, "Economic Load Dispatch Solution using Interval Gradient Method," Advanced Research in Electrical and Electronic Engineering, vol. 1, no. 4, pp. 55-58, 2014.

[14] P. T. Ha, H. M. Hoang, T. T. Nguyen, T. T. Nguyen, "Modified moth swarm algorithm for optimal economic load dispatch problem," TELKOMNIKA Telecommunication Computing Electronics and Control, vol. 18, no. 4, pp. 2140-2147, 2020.

[15] L. H. Pham, T. T. Nguyen, L. D. Pham, N. H. Nguyen, "Stochastic fractal search based method for economic load dispatch," TELKOMNIKA Telecommunication Computing Electronics and Control, vol. 17, no. 5, pp. 2535-2546, 2019.

[16] S. K. Gachhayat, S. K. Dash, P. Ray, "Multi Objective Directed Bee Colony Optimization for Economic Load Dispatch With Enhanced Power Demand and Valve Point Loading," International Journal of Electrical \& Computer Engineering (IJECE), vol. 7, no. 5, pp. 2382-2391, 2017.

[17] S. Mouassa and T. Bouktir, "Artificial bee colony algorithm for solving economic dispatch problems with nonconvex cost functions," International Journal of Power and Energy Conversion, vol. 8, no. 2, pp. 146-165, 2017.

[18] D. S. N. M. Rao, N. Kumar, "Comparisional Investigation of Load Dispatch Solutions with TLBO," International Journal of Electrical and Computer Engineering, vol. 7, no. 6, pp. 3246-3253, 2017.

[19] S. R. Spea, "Solving practical economic load dispatch problem using crow search algorithm," International Journal of Electrical and Computer Engineering, vol. 10, no. 4, pp. 3431-3440, 2020.

[20] K. Rajesh, N. Visali, "Hybrid method for achieving Pareto front on economic emission dispatch," International Journal of Electrical and Computer Engineering (IJECE), vol. 10, no. 4, pp. 3358-3366, 2020.

[21] J. Hetzer, C. Y. David, K. Bhattarai, “An economic dispatch model incorporating wind power," IEEE Transactions on energy conversion, vol. 23, no. 2, pp. 603-611, 2008.

[22] J. T. Jose, "Economic load dispatch including wind power using Bat Algorithm," 2014 International Conference on Advances in Electrical Engineering (ICAEE), Vellore, 2014, pp. 1-4.

[23] W. R. Abdul-Adheem, "An enhanced particle swarm optimization algorithm," International Journal of Electrical and Computer Engineering (IJECE), vol. 9, no. 6, pp. 4904-4907, 2019.

[24] P. Sutyasadi, M. B. Wicaksono, "Joint control of a robotic arm using particle swarm optimization based $\mathrm{H} 2 / \mathrm{H} \infty$ robust control on arduino," TELKOMNIKA Telecommunication Computing Electronics and Control, vol. 18, no. 2, pp. 1021-1029, 2020.

[25] K. Paul, N. Kumar, "Cuckoo Search Algorithm for Congestion Alleviation with Incorporation of Wind Farm," International Journal of Electrical and Computer Engineering (IJECE), vol. 8, no. 6, pp. 4871-4879, 2018.

[26] R. Habachi, A. Boulal, A. Touil, Charkaoui, A. Echchatbi, "Economic and emission dispatch using cuckoo search algorithm," International Journal of Electrical and Computer Engineering (IJECE), vol. 9, no. 5, pp. 3384-3390, 2019.

[27] V. N. Rajput, et al., "A Novel Protection Scheme for Solar Photovoltaic Generator Connected Networks Using Hybrid Harmony Search Algorithm-Bollinger Bands Approach,” Energies, vol. 13, no. 10, p. 2439, 2020.

[28] L. Xiao, M. Dridi, A. Hajjam El Hassani, H. Fei, W. Lin, "An improved cuckoo search for a patient transportation problem with consideration of reducing transport emissions," Sustainability, vol. 10, no. 3, p. 793, 2018.

[29] D. Niu, W. Zhao, S. Li, R. Chen, "Cost Forecasting of Substation Projects Based on Cuckoo Search Algorithm and Support Vector Machines," Sustainability, vol. 10, no. 1, p. 118, 2018.

[30] H. Zhang, D. Yue, X. Xie, C. Dou, F. Sun, "Gradient decent based multi-objective cultural differential evolution for short-term hydrothermal optimal scheduling of economic emission with integrating wind power and photovoltaic power," Energy, vol. 122, pp. 748-766, 2017. 